\title{
Mortalidad por aneurismas aórticos en México: necesidad de un registro nacional
}

\section{Aortic aneurysm-related mortality in Mexico: The need for a national registry}

\author{
Carlos A. Hinojosa ${ }^{1 *}$, Erwin Chiquete ${ }^{2}$ y Luis O. Bobadilla-Rosado ${ }^{1}$ \\ ${ }^{1}$ Departamento de Angiología, Cirugía Vascular y Endovascular; ${ }^{2}$ Departamento de Neurología y Psiquiatría. Instituto Nacional de Ciencias Médicas \\ y Nutrición "Salvador Zubirán”, Ciudad de México, México
}

En las últimas dos décadas, la mortalidad asociada a patologías aórticas (aneurismas rotos y disecciones aórticas) ha pasado de 2.49 a 2.78 por cada 100,000 en 1990 a $2010^{1}$. Resulta interesante que esta se ha reportado mayor en países en desarrollo, con una tasa de 0.71 , en contraste con la tasa de mortalidad de 0.22 reportada en países desarrollados ${ }^{1}$. Así, la mortalidad por ruptura aneurismática se cree que es de hasta un $80 \%$; siendo solo del $30-50 \%$ de estos casos los que alcanzan a tener atención médica ${ }^{2}$. En adición a lo anterior, se ha reportado que al año se pierden más de 2,500,000 años de vida gracias a esta patología². En cuanto a México, Barragán-Galindo, et al. ${ }^{3}$ reportaron, en su revisión de la literatura, 429 pacientes mexicanos intervenidos por aneurisma aórtico (AA) torácico y abdominal, en artículos publicados del 1996 a 2017.

En su análisis de más de 3 millones de pacientes, obtenidos de la cohorte de Life Line Screening, Kent, et al. ${ }^{4}$ encontraron una prevalencia del $0.86 \%$ de $\mathrm{AA}$ en población «hispana», aunque el $87 \%$ de esta cohorte lo comprendían individuos de "raza blanca». En contraste, diversos estudios han reportado prevalencias mayores al $1 \%$ en población latinoamericana; tal es el caso de Betalles, et al. ${ }^{5}$, quienes reportaron una prevalencia del $5.4 \%$ en hombres y del $4.5 \%$ en mujeres entre 45 y 87 años; Souto, et al. ${ }^{6}$ encontraron una prevalencia del $2.5 \%$ en Brasil y García, et al. ${ }^{7}$ reportaron una prevalencia de solo un $0.75 \%$ en Medellín. En México, Enríquez-Vega, et al. ${ }^{8}$ realizaron un estudio prospectivo en 144 pacientes del hospital de especialidades del Instituto Mexicano del Seguro Social, encontrando una prevalencia del $6.9 \%$; igualmente, Hinojosa, et al. ${ }^{9}$ reportaron, en el Instituto Nacional de Ciencias Médicas y Nutrición Salvador Zubirán, que como resultado de un escrutinio ultrasonográfico, hasta el 2018 se había encontrado una prevalencia del 8.4\%. Así, como reportaron Anaya-Ayala, et al. ${ }^{10}$, una posible herramienta para coadyuvar en la detección de esta patología es la búsqueda intencionada de susceptibilidad genética.

En nuestro grupo analizamos las bases de datos abiertas de mortalidad por disección aórtica y AA roto en México desde 1998 al 2012, encontrando una edad promedio de muerte de 69 años (Fig. 1), además, en la fig. 2 podemos observar la incidencia de muertes por año. El presente resultado deja muchas dudas sobre si las muertes secundarias a aneurisma en el país no están siendo subestimadas, tomando en cuenta que solo del 30 al $50 \%$ de los pacientes con esta patología llega a solicitar atención médica a un centro hospitalario ${ }^{2}$, pudiendo existir un subregistro importante en los certificados de defunción. Adicionalmente, al no existir
Correspondencia:

${ }^{*}$ Carlos A. Hinojosa

E-mail: carlos.a.hinojosa@gmail.com

0377-4740/C 2021 Sociedad Mexicana de Angiología y Cirugía Vascular y Endovascular, A.C. Publicado por Permanyer. Este es un artículo open access bajo la licencia CC BY-NC-ND license (http://creativecommons.org/licenses/by-nc-nd/4.0/).

Disponible en internet: 24-09-2021

Rev Mex Angiol. 2021;49(3):71-73 www.RMAngiologia.com
Fecha de recepción: 07-07-2021

Fecha de aceptación: 16-07-2021

Publicado por Permanyer. Este es un artículo open access 


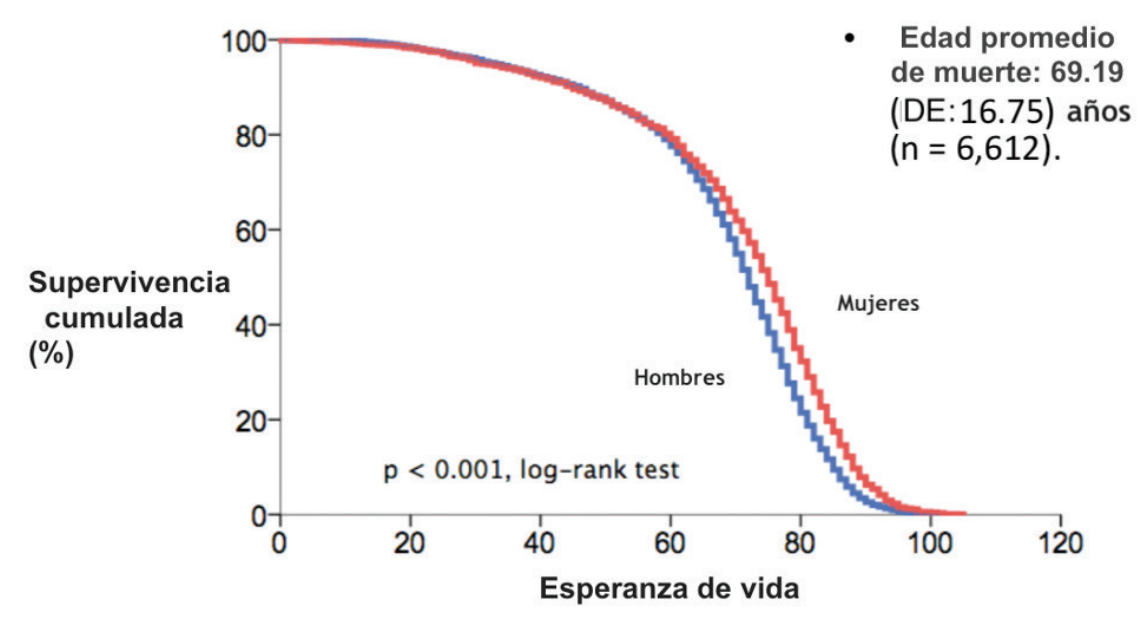

Figura 1. Análisis de la supervivencia en México de pacientes con aneurisma aórtico.

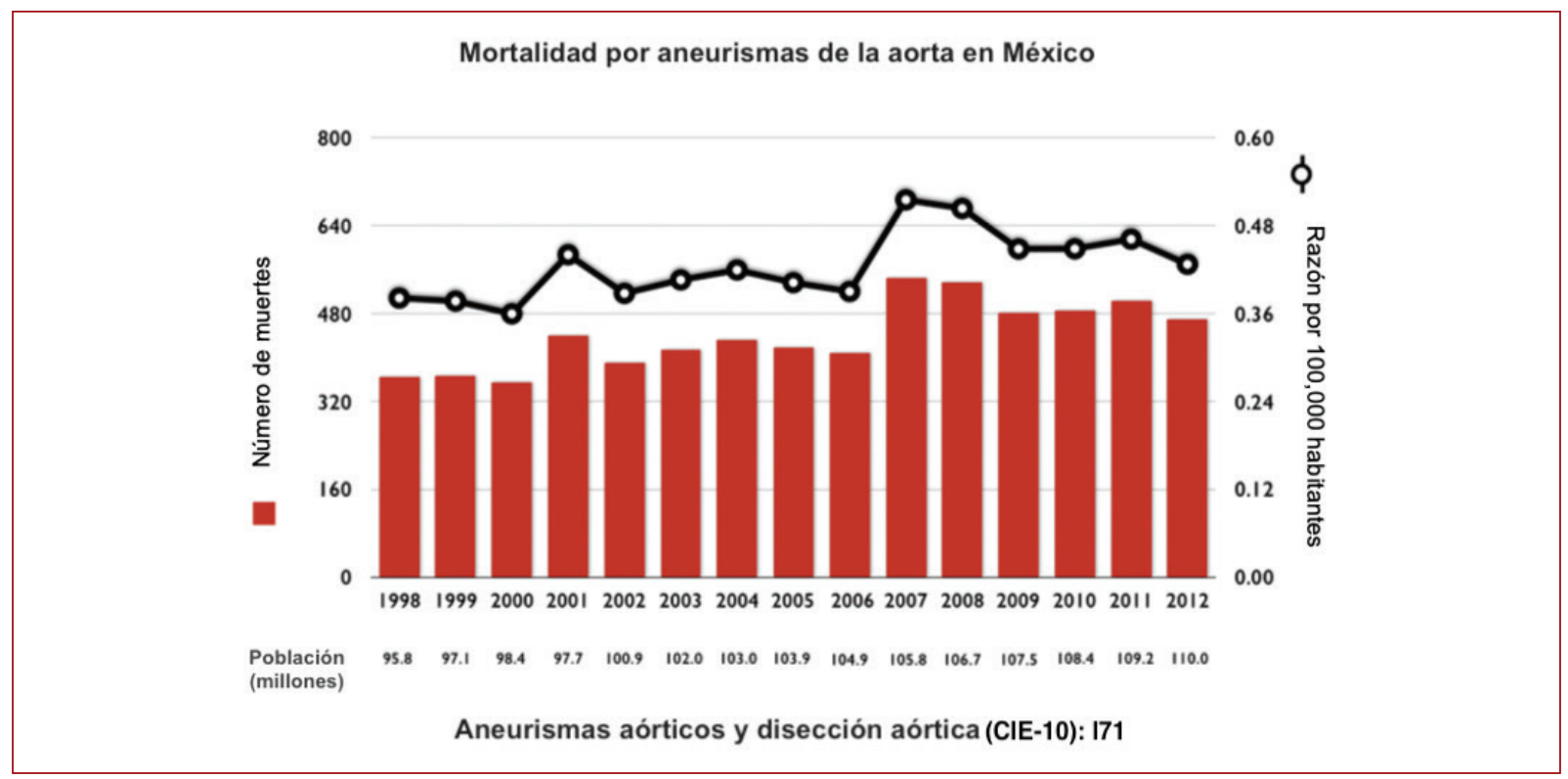

Figura 2. Incidencia de muertes por aneurisma aórtico y disección aórtica en México.

CIE-10: Clasificación Internacional de Enfermedades de la Organización Mundial de la Salud.

un programa de tamizaje de AA en el país, es probable que esta patología sea infradiagnosticada y, con frecuencia, la causa de muerte atribuida a otras patologías consideradas más frecuentes ${ }^{3}$.

De tal forma, debido a que los AA son, en muchos casos, patologías silentes previo a la ruptura, la mejor forma de prevenir muertes secundarias a estos es con una detección activa; por tal motivo, debemos de considerar como prioritario el generar un programa de escrutinio en pacientes mexicanos con factores de riesgo, en adición a un registro nacional, de tal forma que podamos mejorar el entendimiento de esta patología en nuestra población, previniendo la aparición de complicaciones letales y la catástrofe que esto implica para los involucrados.

\section{Bibliografía}

1. Bossone E, Eagle KA. Epidemiology and management of aortic disease:
aortic aneurysms and acute aortic syndromes. Nat Rev Cardiol.
$2021 ; 18(5): 331-48$.
2. Cannon JW. Hemorragic shock. N Eng J Med. 2018;378(4):370-9. 
3. Barragán-Galindo L, Soto-Pérez A, Anaya-Ayala JE, García-Alva $R$, Cuén-Ojeda C, Hinojosa CA. Revisión sistemática de procedimientos quirúrgicos usados para tratar patología aórtica en México. Gac Med Mex. 2019;155:136-42.

4. Kent KC, Zwolak RM, Egorova NN, Riles TS, Manganaro A, Moskowitz AJ, et al. Analysis of risk factors for abdominal aortic aneurysm in a cohort of more than 3 million individuals. J Vasc Surg. 2010 52(3):539-48

5. Maris-Batallés S, Capomasi M, Longo G, Pezzotto SM, Villavivencio R Prevalencia de aneurisma de aorta abdominal evaluada con ecografía aórtica y factores de riesgo cardiovascular. Rev Argent Radiol. 2006; 70:285-8.

6. Souto F, Pontes S, Taylor MA, Roelke L, Sandri J, de Melo Jacques C, et al, Rastreamento do aneurisma da aorta abdominal na populaçao da cidade de Vitória (ES). J Vasc Bras. 2005:4:59-65.
7. García G, Calle W, Ramírez W, Díaz C, Timarán R, Uribe JA, et al. Prevalencia del aneurisma aórtico abdominal en el área urbana de Medellín, Colombia. latreia. 2008;21:S-16.

8. Enríquez-Vega ME, Solorio-Rosete HF, Cossio-Zazueta A, Bizueto-Rosas $\mathrm{H}$, Cruz-Castillo JE, Iturburu-Enríquez A. Detección oportuna de aneurismas de aorta abdominal en población de riesgo. Rev Med Inst Mex Seguro Soc. 2015:53(Suppl 1):S100-3.

9. Hinojosa CA, Bermúdez-Serrato KP, Anaya-Ayala JE, Pérez-Milán R, García-Alva R, Martínez-Méndez G. Las acciones proactivas en la búsqueda de patología aórtica aneurismática tienen un impacto en la prevalencia. Cir Cir. 2019:87:470-6.

10. Anaya-Ayala JE, Hernandez-Doño S, Escamilla-Tilch M, Marquez-Garcia J, Hernandez-Sotelo K, Lozano-Corona R, et al. Genetic polymorphism of HLA-DRB1 alleles in Mexican mestizo patients with abdominal aortic aneurysms. BMC Med Genet. 2019;20(1):102. 